\title{
Comparative Features of Vivax, Falciparum and Mixed Malarial Infections in a Peripheral Hospital of Mumbai.
}

\author{
Dr. Yasmeen Khatib ${ }^{1}$, Dr. Karen Sequeira ${ }^{2}$, Dr.Sanjay Gulhane ${ }^{3}$. \\ ${ }^{1}$ Associate Professor, Dept of Pathology,Dr.R.N.Cooper Municipal General Hospital, Mumbai, India. \\ ${ }^{2}$ Registrar, Dept of Pathology, Dr.R.N.Cooper Municipal General Hospital Hospital, Mumbai, India. \\ ${ }^{3}$ Associate Professor, Dept of Medicine, Dr. R.N.Cooper Municipal General Hospital Hospital, Mumbai, India.
}

\begin{abstract}
Falciparum malaria is known to cause severe malaria with multiorgan involvement while Vivax malaria is thought to run a benign course. However recent studies have reported complications even in vivax malaria and mixed infections. A prospective observational study was conducted for a period of 2 years on all patients who tested positive for malaria by smear examination or antigen test. A comparative evaluation of clinical features diagnostic methods, hematological profile and biochemical profile of patients presenting with vivax falciparum and mixed infection was done..Appropriate statistical analysis was done to compare the various parameters. There were 197 cases of vivax, 76 cases of falciparum and 27 cases of mixed infection out of the total 300 malaria cases. Headache, diarrhoea, vomiting, hepatomegaly, jaundice, anaemia, raised bilirubin, liver enzymes and creatinine were more common in mixed and falciparum infections than in vivax infection while splenomegaly and thrombocytopenia was more common in P. vivax and mixed malaria. Only falciparum cases showed convulsions and acute renal failure, while respiratory distress was seen in both vivax and falciparum infections.Though falciparum and mixed infections present more frequently with complications and altered laboratory parameters even vivax infection can present with severe malaria in less proportion of the cases.
\end{abstract}

Keywords: Biochemical profile, clinical features, hematological parameters, vivax, falcipaum

\section{Introduction}

About 3.2 billion people remain at risk of malaria worldwide. In year 2015 alone there were an estimated 214 million new cases of malaria and 4.38 lakhs deaths. ${ }^{(1)}$ Most of these cases occurred in African region(88\%) followed by South East Asian region(10\%) and Eastern Mediterranean region.(2\%).Malaria in humans can be caused by 4 human species Plasmodium vivax, P.falciparum, P.malariae and P.ovale after a bite of female Anopheles Mosquitoe. ${ }^{(2)}$ P.falciparum and P.vivax pose the greatest public health challenge. P.falciparum is most prevalent in the African continent and is responsible for majority of the deaths due to malaria.P.vivax has a wider geographic distribution because it can develop at even lower temperature, higher altitude and has a dormant liver stage. It accounted for an estimated 13.8 million cases in 2015 and caused $>50 \%$ cases outside Africa.Most cases of vivax malaria occurred in South East Asian Region(74\%) region followed by East Mediterranean region(11\%) and African region.(10\%).More than $80 \%$ cases of vivax occurred in 3 countries Ethopia,India and Pakistan.Though number of deaths due to vivax are estimated to be between 1400 and 14900 globally it causes significant morbidity. In India estimated cases of malaria in 2014 were 1.4 million with 561 deaths. Falciparum contributed to $66 \%$ cases followed $34 \%$ vivax cases. ${ }^{(1)}$ The ability of falciparum infection in causing severe malaria and organ dysfunction is well known.Though vivax infection was considered to be benign recent studies indicate that even it can cause severe malaria in some cases. ${ }^{(3-6)} \mathrm{A}$ considerable number of patients are even presenting with mixed infection. The present study was conducted to do a comparative analysis of clinical presentation, diagnosis, biochemical and hematological profile of patients presenting with vivax falciparum and mixed infections in a peripheral hospital of Mumbai.

\section{Material and Methods}

The present study was a prospective observational study done in the department of pathology at $\mathrm{Dr}$ R.N.Cooper Hospital Mumbai.The duration of the study was a 2 year period between $1^{\text {st }}$ June 2009 till $31^{\text {st }}$ May 2011.Institutional ethics committee permission was obtained. Study included all febrile patients of both sexes who were malaria positive by either thick smear thin smear or antigen detection tests.Thick smears were stained by Jaswant and Bhattacharya Stain and thin smear by fields stain. Smears were examined for malarial parasite in 100 oil immersion fields for at least 5 minutes. A complete clinical history and clinical examination was done with reference to the presence of fever, chills, headcache, vomiting, diarrhoea, oliguria, altered mental status/convulsions, jaundice and hepatosplenomegaly.Complete blood count was done on a fully automated cell counter and biochemical profile was done on a fully automated analyzer.Haemoglobin levels, WBC count and 
platelet count were noted. Serum bilirubin, liver enzymes and serum creatinine levels were noted. A comparative analysis was done among patients with vivax, falciparum and mixed infections.

2.1 Statistical analysis:Statistical analysis was done by SPSS version 16.The data of the 3 groups of vivax falciparum and mixed infection was analysed using the Fisher or Chi square test appropriate for parameters like splenomegaly hepatomegaly along with hematological and biochemical parametes.

\section{Results}

A total of 6366 patients were tested for malarial parasite out of which 300 were positive for malaria and included in the study.There were $197(66 \%)$ cases of vivax,76(25\%) cases of falciparum and $27(9 \%)$ cases of mixed infections. The age and sex distribution is given in Table-1.There were 200 males $(66.67 \%)$ and $100(33.33 \%)$ females with a male female ratio of 2:1.Majority of the cases were in the 21 to $30(31.67 \%)$ years age group followed by 11 to 20(24.33\%) years age group.This trend was seen in vivax and mixed infections while falciparum was more common in 11 to $20(30.26 \%)$ years age group.

3.1 Clinical features. The clinical presentation of patients with vivax, falciparum and mixed infections is given in Table 2. Fever was present in all (100\%) patients followed by chills and rigours in $92.7 \%$ patients and headache in $57 \%$ patients. Chills and rigour was most common in patients with mixed infection $27(100 \%)$ whereas it was similar in patients with P.vivax 179(90.86\%)and P.falciparum 72(94.73\%). Headache was more common in mixed $23(85.2 \%)$ and falciparum malaria48(63\%) than in vivax malaria $101(51 \%)$.Vomitting was seen in $60(20 \%)$ of the cases.It was more frequently observed in mixed(48.2\%) and falciparum $(36.8 \%)$ infections than in vivax $(11.2 \%)$ infection. Diarrhoea was observed in $61(20.33 \%)$ of all the cases.It was more common in mixed infection $48.15 \%$ followed by falciparum $36.84 \%$ and vivax infections $(10.15 \%)$. One case each of falciparum and vivax presented with respiratory distress, while 2 cases of falciparum presented with convulsions.Jaundice was more common in mixed infections $10(37.03 \%)$ than in falciparum14(18.42\%) and vivax malaria $17(8.63 \%)$. Out of the total patients $38(12.66 \%)$ had hepatomegaly and $51(17 \%)$ had splenomegaly. Hepatomegaly was significantly $(\mathrm{p}=<0.0005)$ more common in mixed malaria $(40.74 \%)$ than in falciparum(18.42\%) and vivax infections.(6.6\%).Similarly splenomegaly was also present significantly $(\mathrm{p}=0.015)$ more in mixed infection $(40.74 \%)$ than in vivax $(15.29 \%)$ and falciparum cases $(14.47 \%)$. 3.2Diagnosis.All cases were subjected to thick smear, thin smear and rapid antigen test. 196 cases $(99.49 \%)$ of vivax were positive by thick smear,137 $(69.54 \%)$ by thin smear and $183(92.89 \%)$ by kit method.75 cases (98.68\%)of falciparum were diagnosed by thick smear $45(59.21 \%)$ by thin smear and $70(92.10 \%)$ by kit method.27 cases (100\%)of mixed infection were diagnosed by thick smear and 26(96.29\% )each by thin smear and antigen method.The sesitivity of thin smear in diagnosis of vivax and falciparum malaria was $73.09 \%$ and $69.61 \%$ while the sensitivity of antigen test was $91.03 \%$ and $90.2 \%$.

3.3 Laboratory parameters. Table 3 shows the various laboratory parameters in different Plasmodium species. Anaemia was significantly $(\mathrm{p}=0.003)$ more common in mixed $19(70.4 \%)$ than in falciparum $45(59.2 \%)$ and vivax cases $84(42.6 \%)$. However severe anaemia $(\mathrm{Hb}<7 \mathrm{mg} \%)$ was seen in only $40(13.33 \%)$ cases.Severe anaemia was seen in $25.93 \%$ of mixed infection $17.11 \%$ of falciparum infection and only $10.15 \%$ of vivax infection.Out of 300 patients $226(75 \%)$ had thrombocytopenia.Incidence of thrombocytopenia was significantly $(\mathrm{p}=<.001)$ more in mixed infection $(100 \%)$ than in vivax $(74.10 \%)$ and falciparum $(69.70 \%)$ malaria.Bilirubin was raised $(>1.2 \mathrm{mg})$ in $79(26.3 \%)$ cases. It was significantly $(\mathrm{p}=<.0005)$ higher in mixed malaria $15(55.56 \%)$ and falciparum malaria $26(34.21 \%)$ than in vivax monoinfection. 38(19.29\%) .Liver enymes were raised in 93(31\%) cases.They were increased in 24 cases $(88.88 \%)$ of mixed infection, 27 cases $(35.53 \%)$ of falciparum infections and 42 cases $(21.31 \%$ ) of vivax infections.29 patients $(9.6 \%)$ had raised serum creatinine. High ceratinine levels were seen in were $7(3.55 \%)$ cases of vivax $16(21.05 \%)$ cases of falciparum and $6(22.22 \%)$ cases of mixed infection and the difference was statistically significant. $(\mathrm{p}=<.005)$. Two cases of falciparum had acute renal failure.

\section{Discussion}

A total of 300 malaria positive cases were included in the study.There were $197(66 \%) 0$ cases of vivax $76(25 \%)$ cases of falciparum and $27(9 \%)$ cases of mixed infection. The proportion of vivax and falciparum cases varies in different parts of India. Jadhav et $\mathrm{al}^{(7)}$ conducted a study in Navi Mumbai and found vivax in $44.7 \%$ cases falciparum in $42 \%$ cases and mixed infections in $12.9 \%$ cases.Kochar et al ${ }^{(8)}$ in a study from Bikaner in north western India found $58 \%$ cases of falciparum and $41 \%$ cases of vivax infection. Overall there were 200 males and 100 females positive for malaria with a male female ratio of 2:1 which is similar to studies conducted by Muddaiah et al ${ }^{(5)}$, Mishra et al ${ }^{(9)}$ and Jadhav et al. ${ }^{(7)}$ High infectivity in the male population has been attributed to high mobility and outdoor activities of males as compared to the females. Overall maximum 
cases of malaria were in the 21 to 30 years age group(31.67\%).In a study conducted by Farogh et al ${ }^{(10)}$ and Muddaiah et $\mathrm{al}^{(5)}$ majority patients belonged to 21 to 30 years age group followed by 11 to 20 years as in our study.Maximum cases of vivax 68(34.5\%) and mixed infections 11(40.8\%) were in the 21 to 30 years age group while maximum cases of falciparum 23(30.26\%) were in the 11 to 20 years age group. Symptom analysis showed that fever was the commonest symptom in all the cases followed by chills and rigours in all types of malaria.Headache was most common in mixed infection followed by falciparum and vivax infections. Vomitting and diarrhoea were common in falciparum and mixed infections than in vivax infections.Similar findings have been reported from studies from bundelkhand and Pakistan. ${ }^{(11,12)}$. There were 2 patients presening with oliguria and 2 with convulsions and all had falciparum infection while one patient with vivax presented with respiratory distress.Hence multiorgan involvement is more common in falciparum than vivax as has been reported by other studies $^{(13)}$. Vivax malaria does not have a benign course and this has been reported in several recent studies. ${ }^{(3-}$ $6,14) .17 \%$ of our patients had splenomegaly while $13 \%$ had hepatomegaly. Splenomegaly was more common in mixed and vivax infections than in falciparum infections while hepatomegaly was more common in mixed and falciparum infections than in vivax infections. Similar findings have been reported by a study by Verma et al. ${ }^{(15)}$ Splenomegaly was more common in vivax infections probably because of relapses.Proportion of cases with splenomegaly is very variable in different studies because of differences in immune status of patients in different malaria transmission regions. Anaemia and thrombocytopenia(TCP) are the most frequently found abnormality in patients suffering from malaria ${ }^{(16)}$.

Anaemia was present in $49.33 \%$ of the cases. Adedapo et $\mathrm{al}^{(17)}$ and Ahmed et al ${ }^{(18)}$ have reported anaemia in $43.8 \%$ and $42 \%$ of their cases which is similar to our study.It was more common in falciparum(50\%) and mixed infections $(73.6 \%)$ than in vivax infections(42.13\%).Severe anaemia with haemoglobin $<7$ gm $\%$ was seen more commonly in mixed $(25.93 \%)$ and falciparum $(17.1 \%)$ than in vivax infections(10.2\%).Similar findings have been reported in studies by Singh et $\mathrm{al}^{(11)}$ and Kochar et $\mathrm{al}^{(8)}$. This shows that even vivax can present with severe anaemia and can no longer be considered benign.Severe anaemia may be due to red blood cell destruction, phagocytosis of non parasitized red cells, increased splenic clearance and dyserythropoiesis in the bone marrow. Thrombocytopenia was present in $75 \%$ cases.It was more common in $\operatorname{mixed}(100 \%)$ and in $\operatorname{vivax}(74.1 \%)$ infections than in falciparum $(69.7 \%)$ infections. Faseela et al ${ }^{(19)}$ have reported TCP in $82.77 \%$ cases while Colonel et $\mathrm{al}^{(20)}$ found it in $72 \%$ their of cases.TCP has been reported more frequently in vivax and mixed infections than in falciparum infections by Gupta et $\mathrm{al}^{(21)}$ and Verma et al ${ }^{(21)}$ which is similar to our study. Leucopenia was seen in $13 \%$ cases while leucocytosis was seen in only $2.6 \%$ cases. Jadhav et al $^{(7)}$ found leukopenia in $14 \%$ of their cases and leucocytosis in $4.9 \%$ cases.In our study both were more common in mixed infections.In our study raised bilirubin was seen in $26 \%$ cases while increased liver enzymes were seen in $31 \%$ cases.Bilirubin was raised in only $19.29 \%$ cases of vivax $34.21 \%$ cases of falciparum and $55.56 \%$ cases of mixed infections.Similarly liver enzymes were raised more in mixed infections followed by falciparum and vivax infections. Similar findings have been reported by Limaye et al ${ }^{(6)}$ et al in their study. Increased creatinine levels are seen in falciparum and mixed infections as reported by Piplani et al. ${ }^{(13)}$.ARF was seen only in 2 cases of falciparum in our study while other studies have reported cases even in vivax infection. ${ }^{(14)}$.ARDS was seen in 1 each case of falciparum and vivax infection. Cho Naing et al $^{(22)}$ have reported ARDS in cases of vivax malaria.we had only 2 cases of falciparum with cerebral malaria .Other studies have reported a higher incidence and even in vivax infections ${ }^{(14)}$.Hence though hepatic renal and hematological parameters are more deranged in falciparum and mixed infections they are also seen in vivax infection indicating that vivax infection does not always have a benign course.

4.1 Diagnosis:Thick smear was able to diagnose vivax falciparum and mixed infections in $99.3 \%$ cases.The sensitivity of thin smear was low in both vivax and falciparum infections but high in mixed infections.The sensitivity of rapid antigen test in diagnosis of all three infections was around $90 \%$.Similar results have been found in studies by Parija et $\mathrm{al}^{(23)}$ and Panigrahi et al ${ }^{(24)}$ who reported a sensitivity of thin smear for vivax and falciparum as $69.9 \%$ and $60 \%$.Hence when only thin smear is done one can miss many cases of both vivax and falciparum infections.

\section{Conclusion}

P.vivax is more common than P.falciparum and mixed infections in Mumbai.Though complicated malaria with alterations in haematological and biochemical parameters is more common in falciparum and mixed malaria it can also occur in vivax malaria hence even these patients need to be monitored.

Table-1:Age And Sex Distribution Of Various Species Of Malaria

\begin{tabular}{|l|l|l|l|l|l|l|l|l|l|l|}
\hline $\begin{array}{l}\text { Age } \\
\text { group- } \\
\text { years }\end{array}$ & Males & $\begin{array}{l}\text { Female } \\
\mathrm{s}\end{array}$ & Total & $\%$ & P.vivax & $\%$ & P.falciparum & $\%$ & Mixed infection & $\%$ \\
\hline $0-9$ & 6 & 4 & 10 & 3.33 & 6 & 3.05 & 4 & 5.26 & 0 & 0 \\
\hline
\end{tabular}


Comparative Features Of Vivax, Falciparum And Mixed Malarial Infections In A Peripheral ..

\begin{tabular}{|c|c|c|c|c|c|c|c|c|c|c|}
\hline $10-20$ & 35 & 38 & 73 & 24.33 & 46 & 23.35 & 23 & 30.26 & 4 & 14.8 \\
\hline $21-30$ & 69 & 26 & 95 & 31.67 & 68 & 34.52 & 19 & 25 & 11 & 40.8 \\
\hline $31-40$ & 43 & 14 & 57 & 19 & 40 & 20.30 & 13 & 17.11 & 4 & 14.8 \\
\hline $41-50$ & 19 & 16 & 35 & 11.67 & 24 & 12.18 & 7 & 9.21 & 4 & 14.8 \\
\hline $51-60$ & 17 & 5 & 22 & 7.33 & 10 & 5.07 & 8 & 10.52 & 2 & 7.4 \\
\hline $61-70$ & 6 & 0 & 6 & 2 & 2 & 1.01 & 2 & 1.02 & 2 & 7.4 \\
\hline $71-80$ & 1 & 0 & 1 & 0.33 & 1 & 0.51 & 0 & 0 & 0 & 0 \\
\hline Total & 200 & 100 & 300 & 100 & 197 & & 76 & & 27 & \\
\hline$\% \operatorname{sex}$ & 66.67 & 33.33 & & & & & & & & \\
\hline & \multicolumn{3}{|c|}{ P.vivax } & \multicolumn{3}{|c|}{ P.falciparum } & \multicolumn{4}{|c|}{ Mixed infection } \\
\hline Males & \multicolumn{3}{|c|}{124} & \multicolumn{3}{|c|}{53} & \multicolumn{4}{|c|}{19} \\
\hline Female & \multicolumn{3}{|l|}{72} & \multicolumn{3}{|l|}{23} & \multicolumn{4}{|l|}{8} \\
\hline
\end{tabular}

Table 2: Comparison Of Clinical Features In Different Species Of Malaria

\begin{tabular}{|c|c|c|c|c|}
\hline CLINICAL PRESENTATION & $\begin{array}{c}\text { P.Vivax } \\
(\mathrm{n}=197)\end{array}$ & P.falciparum (n=76) & $\begin{array}{c}\text { Mixed } \\
\text { Infection(n=27) }\end{array}$ & Total \\
\hline FEVER & $197(100 \%)$ & $76(100 \%)$ & $27(100 \%)$ & $300(100 \%)$ \\
\hline CHILLS & $179(90.9 \%)$ & $72(94.7 \%)$ & $27(100 \%)$ & $278(92.7 \%)$ \\
\hline HEADACHE & $101(51.3 \%)$ & $48(63.2 \%)$ & $23(85.2 \%)$ & $172(57.3 \%)$ \\
\hline VOMITING & $22(11.2 \%)$ & $26(36.8 \%)$ & $12(48.2 \%)$ & $60(20 \%)$ \\
\hline DIARRHOEA & $20(10.2 \%)$ & $28(36.8 \%)$ & $13(48.2 \%)$ & $61(20.3 \%)$ \\
\hline OLIGURIA & 0 & $02(0.66 \%)$ & 0 & $02(0.66 \%)$ \\
\hline $\begin{array}{c}\text { CONVSPIRATORY DISTRESS } \\
\text { RENS/NEUROLOGICAL } \\
\text { DEFICIT }\end{array}$ & $01(0.33 \%)$ & $01(0.33 \%)$ & 0 & $02(0.66 \%)$ \\
\hline JAUNDICE & 0 & $02(0.66 \%)$ & 0 & $02(0.66 \%)$ \\
\hline PALLOR & $17(8.6 \%)$ & $14(18.4 \%)$ & $10(37 \%)$ & $41(13.7 \%)$ \\
\hline
\end{tabular}

Table:3 comparison Of Diagnostic Technique In Diagnosis Of Malaria

\begin{tabular}{|l|l|l|l|}
\hline & THICK SMEAR & THIN SMEAR & ANTIGEN TEST \\
\hline PV(P.VIVAX) & $196(99.49 \%)$ & $137(69.54 \%)$ & $183(92.89 \%)$ \\
\hline PF(P.FALCIPARUM) & $75(98.68 \%)$ & $45(59.21 \%)$ & $70(92.10 \%)$ \\
\hline PV,PF & $27(100 \%)$ & $26(96.29 \%)$ & $26(96.29 \%)$ \\
\hline TOTAL & $298(99.33 \%)$ & $208(69 \%)$ & $279(93 \%)$ \\
\hline
\end{tabular}

Table:4 Statistical Analysis Of Various Parameters In Vivax ,Falciparum And Mixed Infections

\begin{tabular}{|l|l|l|l|l|}
\hline PARAMETERS & $\begin{array}{l}\text { P.Vivax } \\
(\mathrm{n}=197)\end{array}$ & P.Falciparum $(\mathrm{n}=76)$ & Mixed infection(n=27) & P value \\
\hline SPLENOMEGALY & $30(15.12 \%)$ & $11(14.5 \%)$ & $10(37 \%)$ & 0.015 \\
\hline HEPATOMEGALY & $13(6.6 \%)$ & $14(18.4 \%)$ & $11(40.7 \%)$ & $<.0005$ \\
\hline ANAEMIA & $84(42.6 \%)$ & $45(59.2 \%)$ & $19(70.4 \%)$ & 0.003 \\
\hline THROMBOCYTOPENIA & $146(74.1 \%)$ & $53(69.7 \%)$ & $27(100 \%)$ & $<.001$ \\
\hline RAISED BILIRUBIN & $38(19.3 \%)$ & $26(34.2 \%)$ & $15(55.6 \%)$ & $<.0005$ \\
\hline RAISED LIVER ENZYMES & $42(21.3 \%)$ & $27(35.5 \%)$ & $24(88.9 \%)$ & $<.0005$ \\
\hline RAISED CREATININE & $7(3.6 \%)$ & $16(21.1 \%)$ & $6(22.2 \%)$ & $<.0005$ \\
\hline
\end{tabular}

$\mathrm{N}=$ total no of cases $\mathrm{p}$ value $-<0.05$ is significant

\section{References}

[1]. World Malaria Report, 2015,Geneva Switzerland:WHO press:2015

[2]. Kumar A, Valecha N, Jain T, Dash A P. Burden of Malaria in India: Retrospective and Prospective View. Am J Trop Med Hyg 2007; 77(S6):69-78.

[3]. Abdallah TM, Abdeen MT, Ahmed IS, Hamdan HZ, Magzoub M, Adam I; Severe Plasmodium falciparum and Plasmodium vivax malaria among adults at Kassala Hospital, eastern Sudan. Malar J 2013;12:148.

[4]. Vinayak V Shelat, Harshal T Pandve, Gayatri Pathak; Socio-demographic characters, clinical profile and laboratory parameters in malaria cases due Plasmodium falciparum and Plasmodium vivax: A comparative study. Journal of Medicine in the Tropics 2014; 16:2:76-80

[5]. Muddaiah M, Prakash PS; A study of clinical profile of malaria in a tertiary referral centre in South Canara. J Vector Borne Dis 2006; 43:29-33

[6]. Limaye CS, Londhey VA, Nabar ST; The study of complications of vivax malaria in comparision with falciparum malaria in Mumbai. J Assoc Physicians India 2012; 60: 15-18.

[7]. Jadhav UM, Patkar VS, kadam NN; Thhrombocytopenia in malaria - co relation with type and severity of malaria. J Assoc Physicians India. 2004; 52:615.

[8]. D. K. Kochar, A. Das, S. K. Kochar; -Severe Plasmodium vivax malaria: a report on serial cases from Bikaner in northwestern India, American Journal of Tropical Medicine and Hygiene, 2009; 80(2):194-198.

[9]. Mishra G; Hospital based study of malaria in Ratnagiri district, Maharashtra. J Vector Borne Dis 2003; 40:109-11

[10]. Farogh A, Quaaum A, aamir haleem, Ghaffar A; Haematological abnormalities in malaria. Biomedica 2009; 25: 52-55

[11]. Neeshu S, Mathur A, Naim F, Agarwal N; The changing clinical spectrum of Malaria: a clinical study from Bundelkhand. IOSR journal of Dental and Medical Sciences 2014; 13(6) 37-40. 19.

[12]. Singh J, Bhargav P,.Beg MA, Sani N, Mehraj V, Jafri W, et al. Comparative features and outcomes of malaria at a tertiary care hospital in Karachi, Pakistan. Int J Infect Dis. 2008 Jan;12(1):37-42. 
[13]. Piplani S; Clinical study of Falciparum malaria in Notheast. JAPI 2000; 48(1):110.

[14]. A Comparative Study of Clinical Profiles of Vivax and Falciparum Malaria in Children at a Tertiary Care Centre in Uttarakhand Ragini Singh, Shruti Kumar, S.K. Rana, Brijesh Thakur, S.P. Singh.2013:7(10)2234-37.

[15]. A Study ON Clinical Presentation and Outcome of Malaria from an Underreported, P.vivax Predominant Region of North India. VermaRK, Richa G, Singh N, VermaS, SrivastavV, Sch. J. App. Med. Sci., 2016; 4(1C):233-243.

[16]. Davis TM, Krishna S, Loopreesuwan \& Supanaranond W, Pukruttayakamee s, Attatamsooonthorn \& white NJ. Erythrocyte sequestration and anemia in severe falciparum malaria. Analysis of acute changes in venous hematocrit using a simple mathematical model. j clin Invest 1990;86(3)793-800

[17]. Adedapo AD, Falade CO, Kotila RT, Ademowo GO. Age as a risk factor for thrombocytopenia and anemia in children treated fpr acute uncomplicated falciparum malaria. J Vector Borne Dis. 2007 Dec;44(4):266-71

[18]. Ahmed S, Adil F, Shahzad T, Yahiya Y. Severe malaria in children: factors predictive of outcome and response to Quinine. J Pak Med Assoc. 2011 Jan;61(1):54-58

[19]. Faseela TS, Roche R, Anita KB, Malli C, Rai Y. Diagnostic Value of Platelet count in Malaria. Journal of Clinical and Diagnostic Research. 2011; 5(3):464-6

[20]. Colonel KM, Bhika RD, Khalid S, Khalique-ur-Rehman S, Syes ZA. Severe thrombocytopenia and prolonged bleeding time in patients with malaria (a clinical study of 162 malaria cases). World Appl Sci J 2010;9:484-8

[21]. Narendra Kumar Gupta, ShyamBabuBansal, Uttam Chand Jain, Kiran Sahare; Study of thrombocytopenia in patients of malaria. Tropical parasitology. 2013;3(1):58-61.

[22]. Cho Naing, Maxine A. Whittaker, Victor Nyunt Wai, Joon Wah Mak; Is Plasmodium vivax Malaria a Severe Malaria?: A Systematic Review and Meta-Analysis. Plos Negl Trop Dis. 2014; 8(8): e3071.

[23]. Parija SC, Dhodapkar R, Elangovan S, Chaya DR. A comparative study of blood smear, QBC and antigen detection for the diagnosis of malaria. 2009;52(2):200-202.

[24]. Panigrahi K. A coparative study of peripheral blood smear,QBC and Antigen detection test in Diagnosis of Malaria,in a tertiary care hospital.IJRRMS.2013;3(3)35-38. 